\title{
INFECTIOUS DISEASES, NSW: DECEMBER 1998
}

In recent weeks there has been an increase in reports of Ross River virus infections, particularly from rural Macquarie and Greater Murray Areas (Figure 1). The increase is earlier than expected for the season, and has prompted local public warnings about the importance of personal protection to avoid infection through being bitten by mosquitoes. These measures include screening external windows and doors in houses, removing stagnant water from around the house, covering up with loosefitting long sleeves and trousers and using plenty of insect repellent, especially when going outdoors around and soon after dusk.

Reports of pertussis have been on the rise since June.
Many of these cases have occurred in the Greater Murray, Southern NSW and Hunter areas (Table 3). These reports highlight the need for health care providers to be vigilant in checking the immunisation status of all children attending for any reason, and to have a high index of suspicion for this infection in patients of any age attending with a coughing illness. Suspected cases should be notified (confidentially) to the local public health unit (PHU). While treatment has little impact on symptoms (except if given very early in the course of the infection), it is effective in stopping transmission to contacts. PHU staff can help arrange preventive therapy for close contacts of cases.

\section{YEAR IN REVIEW INFECTIOUS DISEASES SURVEILLANCE: 1997}

In this issue, we provide a summary of infectious disease surveillance for 1997. Tables 4 through 7 provide breakdowns of the notifiable diseases for NSW by year, month, area of residence, age group and sex.

\section{DISEASES WITH INCREASED NOTIFICATIONS}

There were 1610 cases of Ross River virus infection reported by laboratories. This was a significant increase over previous years and probably relates to both an increased prevalence of infected mosquitoes, and better recognition of the disease by laboratories, doctors, and the public. The epidemic affected most rural areas of the state, and large numbers of cases were reported from the Greater Murray, Hunter, Central Coast, Illawarra and Far West areas. Some cases were also reported that had resulted from exposure to mosquitoes in bushland areas on the periphery of Sydney.

Reports of gonorrhoea continued to increase in 1997, particularly in young inner city men, highlighting the need for a continuing emphasis on the practice of safe sex.

Hepatitis A reports also jumped dramatically in 1997. Several hundred of these cases were attributable to an outbreak traced to eating contaminated oysters in the early part of the year. ${ }^{1}$ A smaller outbreak of 23 cases was traced to consumption of contaminated prawns at a Sydney restaurant in May. ${ }^{2}$ Other cases were attributable to personto-person spread, notably among young men in the inner city. These latter cases are a reminder of the value of hepatitis A vaccination for gay men and other high risk groups.

Laboratory reports of positive antibodies to hepatitis $\mathbf{C}$ virus remained the most commonly reported condition, with almost 9000 case reports in 1997 . However, many of these reports are likely to represent persons infected in previous years. On the other hand, many persons with new hepatitis $\mathrm{C}$ infections have no symptoms, do not seek testing and, so, do not appear in these surveillance data.

There were 222 cases of meningococcal disease in 1997. An unusually high proportion of cases were adolescents or young adults, which may be explained in part by the emergence of a relatively new strain of serogroup $C$ in the mid 1990s. (There is some evidence that the appearance of a new strain leads to an upward shift in the age groups of cases in affected communities.) Three small clusters of illness due to serogroup C were identified in 1997. These included three students at a university in August, ${ }^{3}$ and three students at a high school in October. ${ }^{4}$ Both of these 
clusters led to vaccination programs at the respective institutions. The third cluster was two students (including one from out-of-state) who attended a university rowing carnival.

There were 4336 cases of pertussis (including the deaths of four infants) reported with onset in 1997, more than twice the number reported for any year since 1991. Cases occurred across all areas of the state, and many were school-aged children or young adults.

\section{DISEASES WITH DECREASED NOTIFICATION}

Some of the best news for years has been generated by the use of highly active antiretroviral therapy against HIV infection. While the number of new reports of HIV infection have remained relatively stable — at around 400 per year-since 1994, the number of new AIDS diagnoses has more than halved in the past few years, most likely due to the availability of these new treatments.

Reports of invasive Haemophilus influenzae type b infections (formerly a major cause of meningitis in infants) remained well down on historical levels since the introduction of vaccine for small children in 1993. Interestingly, only eight of the 18 cases reported in 1997 were younger than five years of age.

\section{Thank you}

Behind each case that contributes to these surveillance statistics lies a person with an illness requiring medical attention. The surveillance system is the backbone of disease control in NSW; for it to work well requires the good will and enthusiasm of patients (who are usually keen to talk to PHU staff to find out what might have contributed to their illness), their doctors and laboratories. Notification of an illness is confidential and required by law.

To all those health care workers and laboratory staff who contribute to disease surveillance and control by notifying cases, helping in case follow-up or arranging preventive therapy, we salute you!

\section{REFERENCES}

1. NSW Health Department. Infectious Diseases Report. NSW Public Health Bulletin 1997; 8: 1,5

2. NSW Health Department. Infectious Diseases Report. NSW Public Health Bulletin 1997; 8: 51

3. NSW Health Department. Infectious Diseases Report. NSW Public Health Bulletin 1997; 8: 69

4. NSW Health Department. Infectious Diseases Report. NSW Public Health Bulletin 1997; 8: 86 


\section{FIGURE 1}

REPORTS OF SELECTED INFECTIOUS DISEASES, NSW, JANUARY 1994 TO NOVEMBER 1998, BY MONTH OF ONSET

These are preliminary data: case counts in recent months may increase because of reporting delays
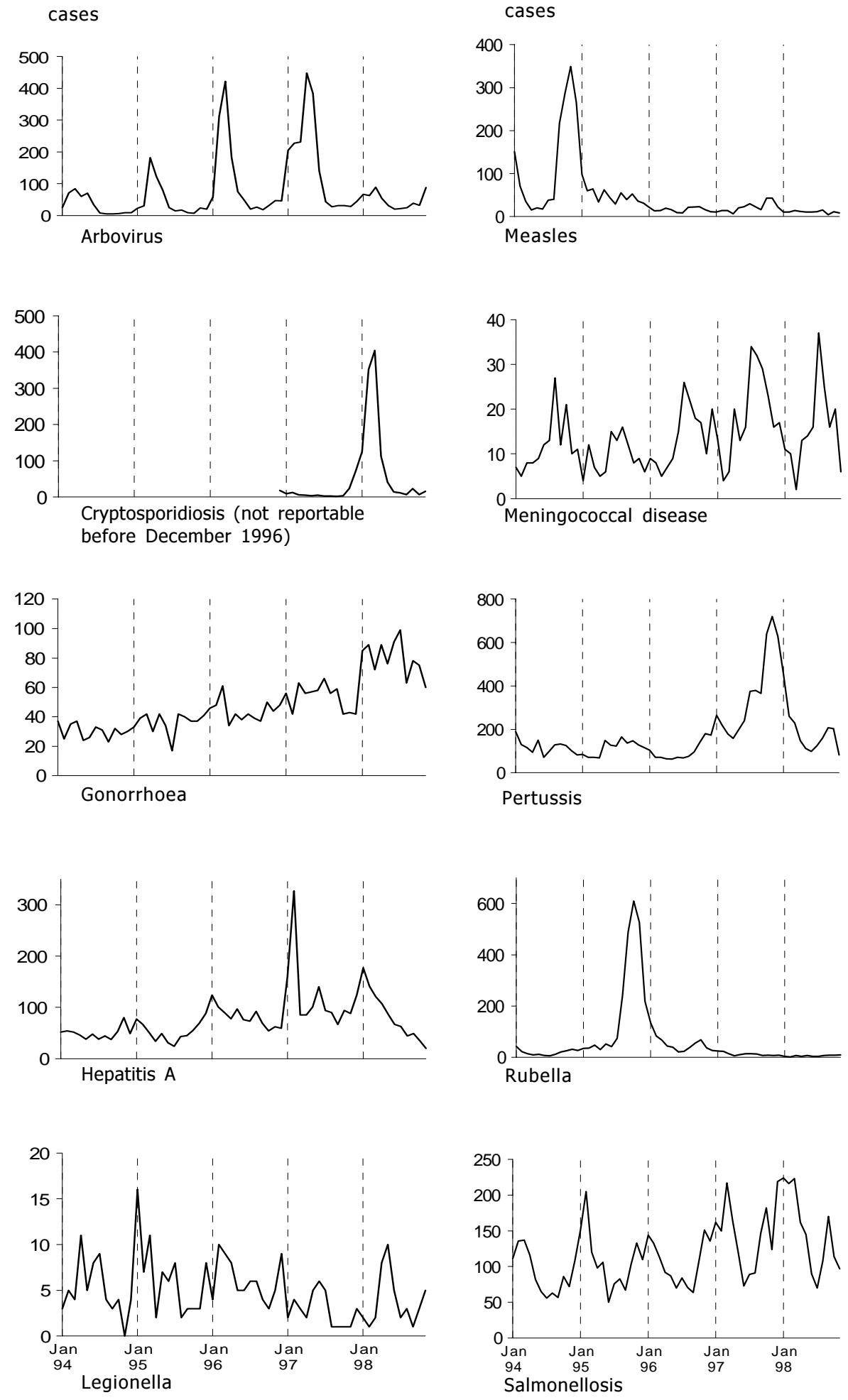


\section{Condition}

CSA NSA

WSA WEN SWS

CCA HUN Area Health Service (1998)

Blood-borne and sexually transmitted

AIDS

Hepatitis B: acute viral

Hepatitis D: unspecified*

Hepatitis, acute viral (not otherwise specified)

Chlamydia (genital)

Syphilis

Vector-borne

Arboviral infection*

Malaria*

Zoonoses

Beptospirosis*

Q fever*

Respiratory and othe

Blood lead leve

Legionnaires' disease

Leprosy

Meningococcal infection (invasive)

Mycobacterial tuberculosis
Mycobacteria other than TB

$\begin{array}{rrrrrrrrr}6 & 5 & 1 & 1 & - & - & - & 3 \\ 1 & 1 & 2 & 2 & - & - & - & - \\ - & - & - & - & - & - & - & - \\ 54 & 28 & - & 2 & 4 & 9 & 7 & 7 & - \\ 2 & 1 & - & - & - & - & - & - & \\ 64 & 55 & 3 & 42 & - & 57 & 69 & - \\ - & - & - & - & - & - & - & - \\ - & - & - & - & - & - & - & - & \\ 10 & 6 & - & 5 & - & - & 6 & 17 & 296 \\ 10 & 3 & - & - & - & 2 & 2 & 2 & 7 \\ 8 & 2 & - & 1 & - & - & - & - & \end{array}$

II SES

NRA MNC

NEA MAC MNA FWA GMA SA

Total
for Nov**To datef

Vaccine-preventable

Adverse event after immunisation

Haemophilus influenzae b infection (invasive)

Mumps*

Pertussis

Rubella*

Tetanus

\section{Faecal-oral}

Botulism

Cryptosporidiosis

Giardiasis

Food borne illness (not otherwise specified)

Gastroenteritis (in an institution)

Haemolytic uraemic syndrome

Hepatitis A

Hepatitis E

Salmonellosis (not otherwise specified)*

Typhoid and paratyphoid*

Verotoxin-producing $\mathrm{E}$ coll

* laboratory-confirmed cases onlył includes cases with unknown postcode

\section{CSA $=$ Central Sydney Area}

NSA $=$ Northern Sydney Area

WEN $=$ Wentworth Area

WSA $=$ Western Sydney Area 


\section{TABLE 4}

\section{DISEASE NOTIFICATIONS IN NSW, 1991 TO 1997}

\begin{tabular}{|c|c|c|c|c|c|c|c|}
\hline \multirow[b]{2}{*}{ Condition } & \multicolumn{7}{|c|}{ Year of Onset } \\
\hline & 1991 & 1992 & 1993 & 1994 & 1995 & 1996 & 1997 \\
\hline Adverse event after immunisation & NN & 31 & 24 & 27 & 28 & 23 & 50 \\
\hline AIDS & 440 & 439 & 483 & 538 & 450 & 341 & 163 \\
\hline Arboviral: Barmah virus infections* & 6 & 6 & 25 & 41 & 280 & 178 & 190 \\
\hline Arboviral: Ross River virus infections* & 305 & 329 & 596 & 333 & 241 & 1048 & 1610 \\
\hline Arboviral: Other Infections* & 118 & 12 & 33 & 11 & 28 & 29 & 35 \\
\hline Blood lead level $>=15 \mathrm{ug} / \mathrm{dl}$ & \multicolumn{5}{|c|}{ not notifiable until December 1996} & 227 & 653 \\
\hline Brucellosis* & 2 & 2 & 4 & 4 & 2 & 1 & 3 \\
\hline Cholera* & 1 & 0 & 1 & 0 & 1 & 4 & 1 \\
\hline Cryptosporidiosis* & \multicolumn{5}{|c|}{ not notifiable until December 1996} & 23 & 159 \\
\hline Diptheria & 0 & 0 & 0 & 0 & 0 & 0 & 0 \\
\hline Food-borne illness (NOS) & 2342 & 228 & 115 & 221 & 288 & 218 & 272 \\
\hline Gastroenteritis (in an institution) & 166 & 411 & 437 & 305 & 1371 & 564 & 946 \\
\hline Gonorrhoea* & 403 & 494 & 387 & 361 & 431 & 526 & 639 \\
\hline Invasive $H$. influenzae type $\mathrm{b}$ infections (total) & 240 & 223 & 126 & 61 & 30 & 14 & 18 \\
\hline H. influenzae type b epiglottitis & 17 & 58 & 32 & 21 & 6 & 2 & 5 \\
\hline H. influenzae type b meningitis & 51 & 106 & 55 & 17 & 11 & 4 & 3 \\
\hline H. influenzae type b septicaemia & 11 & 27 & 24 & 12 & 9 & 3 & 1 \\
\hline H. influenzae type b infection (NOS) & 161 & 32 & 15 & 11 & 4 & 5 & 9 \\
\hline Haemolytic uraemic syndrome & \multicolumn{5}{|c|}{ not notifiable until December 1996} & 0 & 3 \\
\hline Hepatitis A* & 1182 & 921 & 593 & 591 & 629 & 971 & 1455 \\
\hline Hepatitis B: acute viral* & 431 & 121 & 102 & 79 & 66 & 44 & 50 \\
\hline Hepatitis B: chronic or carrier* & 44 & 603 & 807 & 927 & 834 & 1212 & 1245 \\
\hline Hepatitis B: unspecified* & 1173 & 2762 & 3126 & 3625 & 3956 & 3114 & 2818 \\
\hline Hepatitis C: acute viral* & 25 & 30 & 31 & 24 & 38 & 22 & 20 \\
\hline Hepatitis C: unspecified* & 990 & 4251 & 6618 & 9256 & 8264 & 8634 & 8942 \\
\hline Hepatitis D: acute viral* & 0 & 0 & 0 & 1 & 2 & 1 & 3 \\
\hline Hepatitis D: unspecified* & 0 & 8 & 13 & 19 & 18 & 8 & 9 \\
\hline Hepatitis E* & 0 & 0 & 1 & 2 & 0 & 3 & 6 \\
\hline Hepatitis: acute viral (NOS) & 61 & 16 & 6 & 2 & 2 & 3 & 1 \\
\hline HIV infection* & 788 & 638 & 517 & 430 & 435 & 412 & 398 \\
\hline Legionnaires' disease* & 39 & 105 & 67 & 60 & 75 & 74 & 33 \\
\hline Leprosy & 1 & 8 & 5 & 3 & 3 & 2 & 0 \\
\hline Leptospirosis* & 31 & 21 & 16 & 14 & 6 & 33 & 34 \\
\hline Listeriosis* & 11 & 14 & 13 & 10 & 14 & 22 & 23 \\
\hline Malaria* & 202 & 164 & 164 & 187 & 206 & 233 & 192 \\
\hline Measles & 540 & 816 & 2372 & 1501 & 604 & 193 & 264 \\
\hline Meningococcal infections (total) & 138 & 123 & 153 & 143 & 113 & 165 & 222 \\
\hline Meningococcal meningitis & 58 & 95 & 98 & 81 & 72 & 102 & 110 \\
\hline Meningococcal septicaemia & 18 & 18 & 43 & 41 & 26 & 40 & 65 \\
\hline Meningococcal infection (NOS) & 62 & 10 & 12 & 21 & 15 & 23 & 47 \\
\hline Mumps* & 8 & 23 & 13 & 11 & 14 & 27 & 29 \\
\hline Mycobacterial infection: other than TB* & 330 & 410 & 481 & 553 & 507 & 453 & 371 \\
\hline Mycobacterial tuberculosis & 461 & 394 & 396 & 400 & 454 & 412 & 461 \\
\hline Pertussis & 55 & 221 & 1546 & 1420 & 1390 & 1175 & 4336 \\
\hline Poliomyelitis & 0 & 0 & 0 & 0 & 0 & 0 & 0 \\
\hline Q Fever* & 173 & 213 & 406 & 268 & 206 & 296 & 265 \\
\hline Rubella* & 64 & 328 & 1187 & 230 & 2391 & 636 & 155 \\
\hline Rubella (Congenital)* & 1 & 0 & 2 & 4 & 1 & 5 & 0 \\
\hline Salmonella infections (total)* & 1302 & 827 & 1002 & 1126 & 1405 & 1259 & 1732 \\
\hline Salmonella bovis morbificans infections* & 20 & 21 & 31 & 23 & 17 & 11 & 27 \\
\hline Salmonella typhimurium infections* & 216 & 238 & 297 & 465 & 558 & 597 & 950 \\
\hline Salmonella infections (NOS)* & 1066 & 568 & 674 & 638 & 830 & 651 & 755 \\
\hline Syphilis & 642 & 933 & 781 & 1063 & 903 & 730 & 571 \\
\hline Tetanus & 6 & 2 & 5 & 4 & 0 & 1 & 3 \\
\hline Typhoid and paratyphoid* & 59 & 28 & 37 & 36 & 40 & 48 & 33 \\
\hline Viral haemorrhagic fever & 0 & 0 & 0 & 0 & 0 & 0 & 0 \\
\hline * Laboratory-confirmed cases only & t ot & Spe & & $=\mathrm{N}$ & able & & \\
\hline
\end{tabular}


TABLE 5

DISEASE NOTIFICATIONS, BY PUBLIC HEALTH UNIT AREA, NSW, 1997

\begin{tabular}{|c|c|c|c|c|c|c|c|c|c|}
\hline Conditions & $\mathrm{CCA}$ & CSA & FWA & GMA & HUN & ILL & MAC & MNC & MWA \\
\hline Adverse event after immunisation & 0 & 0 & 0 & 10 & 0 & 0 & 0 & 1 & 3 \\
\hline AIDS & 1 & 29 & 1 & 1 & 7 & 3 & 0 & 3 & 0 \\
\hline Arboviral: Barmah virus infections* & 0 & 1 & 5 & 9 & 4 & 7 & 2 & 99 & 2 \\
\hline Arboviral: Ross river virus infections* & 191 & 16 & 103 & 299 & 249 & 109 & 82 & 88 & 48 \\
\hline Arboviral: Other Infections* & 0 & 2 & 0 & 3 & 4 & 3 & 0 & 7 & 0 \\
\hline Blood lead level $>=15 \mathrm{ug} / \mathrm{dl}$ & 6 & 12 & 1 & 11 & 354 & 28 & 4 & 2 & 3 \\
\hline Brucellosis* & 0 & 1 & 0 & 0 & 0 & 0 & 0 & 1 & 0 \\
\hline Cholera* & 0 & 0 & 0 & 0 & 0 & 0 & 0 & 0 & 0 \\
\hline Cryptosporidiosis* & 0 & 8 & 1 & 11 & 4 & 2 & 9 & 29 & 1 \\
\hline Food-borne illness (NOS) & 73 & 87 & 18 & 17 & 0 & 0 & 5 & 0 & 5 \\
\hline Gastroenteritis (institution) & 93 & 56 & 3 & 0 & 367 & 0 & 4 & 0 & 0 \\
\hline Gonorrhoea* & 7 & 101 & 4 & 3 & 19 & 8 & 17 & 11 & 3 \\
\hline Invasive $H$.influenzae type $\mathrm{b}$ infections (total) & 1 & 0 & 0 & 0 & 1 & 2 & 0 & 0 & 0 \\
\hline H.influenzae type b epiglottitis & 0 & 0 & 0 & 0 & 0 & 0 & 0 & 0 & 0 \\
\hline H.influenzae type b meningitis & 0 & 0 & 0 & 0 & 0 & 0 & 0 & 0 & 0 \\
\hline H.influenzae type b septicaemia & 0 & 0 & 0 & 0 & 0 & 0 & 0 & 0 & 0 \\
\hline H.influenzae type b infection (NOS) & 1 & 0 & 0 & 0 & 1 & 2 & 0 & 0 & 0 \\
\hline Haemolytic uraemic syndrome & 0 & 0 & 0 & 0 & 0 & 0 & 0 & 1 & 1 \\
\hline Hepatitis $A^{*}$ & 43 & 127 & 53 & 12 & 121 & 34 & 52 & 91 & 54 \\
\hline Hepatitis B: acute viral* & 2 & 4 & 1 & 1 & 3 & 0 & 0 & 3 & 1 \\
\hline Hepatitis B: chronic or carrier* & 9 & 10 & 5 & 13 & 26 & 0 & 1 & 8 & 29 \\
\hline Hepatitis B: unspecified* & 60 & 665 & 7 & 16 & 44 & 95 & 16 & 15 & 24 \\
\hline Hepatitis C: acute viral* & 0 & 2 & 1 & 0 & 1 & 0 & 1 & 1 & 0 \\
\hline Hepatitis C: unspecified* & 370 & 864 & 28 & 179 & 515 & 351 & 75 & 212 & 319 \\
\hline Hepatitis D: acute viral* & 0 & 0 & 0 & 0 & 1 & 0 & 0 & 0 & 2 \\
\hline Hepatitis D: unspecified* & 0 & 0 & 0 & 0 & 1 & 0 & 0 & 0 & 0 \\
\hline Hepatitis E* & 1 & 1 & 0 & 0 & 0 & 0 & 0 & 0 & 0 \\
\hline Hepatitis: acute viral (NOS) & 0 & 0 & 0 & 0 & 0 & 0 & 0 & 0 & 0 \\
\hline HIV infection* & 2 & 61 & 0 & 0 & 16 & 8 & 0 & 5 & 3 \\
\hline Legionnaires' disease* & 0 & 8 & 0 & 0 & 5 & 4 & 0 & 1 & 1 \\
\hline Leptospirosis* & 1 & 0 & 0 & 3 & 11 & 0 & 0 & 1 & 1 \\
\hline Listeriosis* & 2 & 2 & 0 & 1 & 2 & 1 & 0 & 1 & 1 \\
\hline Malaria* & 4 & 21 & 0 & 3 & 7 & 3 & 4 & 3 & 1 \\
\hline Measles & 8 & 15 & 2 & 4 & 46 & 11 & 10 & 9 & 9 \\
\hline Meningococcal infections (total) & 15 & 18 & 3 & 3 & 14 & 13 & 6 & 7 & 13 \\
\hline Meningococcal meningitis & 10 & 11 & 1 & 0 & 8 & 10 & 3 & 3 & 8 \\
\hline Meningococcal septicaemia & 0 & 6 & 0 & 1 & 3 & 2 & 0 & 2 & 3 \\
\hline Meningococcal infection NOS) & 5 & 1 & 2 & 2 & 3 & 1 & 3 & 2 & 2 \\
\hline Mumps* & 1 & 4 & 8 & 0 & 1 & 0 & 0 & 0 & 0 \\
\hline Mycobacterial infection: other than TB* & 13 & 63 & 0 & 8 & 30 & 2 & 5 & 13 & 0 \\
\hline Mycobacterial tuberculosis & 2 & 80 & 2 & 3 & 16 & 14 & 1 & 8 & 7 \\
\hline Pertussis & 80 & 298 & 30 & 67 & 688 & 190 & 41 & 123 & 84 \\
\hline Q Fever* & 4 & 1 & 13 & 30 & 9 & 2 & 51 & 41 & 23 \\
\hline Rubella* & 6 & 9 & 1 & 3 & 14 & 4 & 2 & 5 & 3 \\
\hline Salmonella infections (total)* & 62 & 112 & 26 & 48 & 145 & 57 & 29 & 67 & 43 \\
\hline Salmonella bovis morbificans infections* & 0 & 2 & 1 & 0 & 0 & 1 & 1 & 3 & 0 \\
\hline Salmonella typhimurium infections* & 25 & 74 & 6 & 28 & 88 & 35 & 5 & 27 & 18 \\
\hline Salmonella infections (NOS)* & 37 & 36 & 19 & 20 & 57 & 21 & 23 & 37 & 25 \\
\hline Syphilis & 7 & 104 & 24 & 3 & 12 & 5 & 25 & 11 & 12 \\
\hline Tetanus & 0 & 0 & 0 & 0 & 2 & 0 & 0 & 0 & 0 \\
\hline Typhoid and paratyphoid* & 0 & 6 & 0 & 2 & 1 & 0 & 0 & 0 & 1 \\
\hline
\end{tabular}

* lab-confirmed cases only NOS = Not Otherwise Specified

Area health service population estimates 1997:
$\mathrm{CCA}=$ Central Coast Area
(279 475)
$\mathrm{CSA}=$ Central Sydney Area
(457 702)
FWA $=$ Far West Area
(52 596)
GMA $=$ Greater Murray Area
(264 083)
HUN = Hunter Area
(540 242)
ILL = Illawarra Area
(342 190)
$\mathrm{MAC}=$ Macquarie Area
(107 033)
MNC = Mid North Coast Area
(256 634)
MWA = Mid Western Area
(172 366) 


\section{TABLE 5}

\section{DISEASE NOTIFICATIONS BY PUBLIC HEALTH UNIT AREA, NSW, 1997 continued}

\begin{tabular}{|c|c|c|c|c|c|c|c|c|c|}
\hline Conditions & NEA & NRA & NSA & SA & SES & SWS & WEN & WSA & NOS \\
\hline Adverse event after immunisation & 0 & 0 & 0 & 1 & 18 & 1 & 7 & 9 & 0 \\
\hline AIDS & 1 & 3 & 20 & 2 & 66 & 10 & 2 & 14 & 0 \\
\hline Arboviral: Barmah virus infections* & 6 & 41 & 3 & 2 & 6 & 0 & 1 & 1 & 1 \\
\hline Arboviral: Ross river virus infections* & 40 & 67 & 69 & 28 & 29 & 24 & 82 & 39 & 47 \\
\hline Arboviral: Other Infections* & 0 & 0 & 6 & 0 & 5 & 3 & 0 & 2 & 0 \\
\hline Blood lead level $>=15 \mathrm{ug} / \mathrm{dl}$ & 10 & 11 & 9 & 6 & 28 & 97 & 28 & 43 & 0 \\
\hline Brucellosis* & 0 & 0 & 0 & 0 & 1 & 0 & 0 & 0 & 0 \\
\hline Cholera* & 0 & 0 & 0 & 0 & 1 & 0 & 0 & 0 & 0 \\
\hline Cryptosporidiosis* & 39 & 18 & 0 & 5 & 9 & 3 & 10 & 10 & 0 \\
\hline Food-borne illness (NOS) & 0 & 3 & 8 & 9 & 14 & 30 & 0 & 2 & 1 \\
\hline Gastroenteritis (institution) & 0 & 0 & 10 & 0 & 319 & 51 & 8 & 6 & 29 \\
\hline Gonorrhoea* & 16 & 14 & 40 & 1 & 295 & 30 & 8 & 43 & 19 \\
\hline Invasive $H$.influenzae type $b$ infections (total) & 0 & 0 & 0 & 3 & 2 & 4 & 4 & 1 & 0 \\
\hline H.influenzae type b epiglottitis & 0 & 0 & 0 & 1 & 0 & 1 & 2 & 1 & 0 \\
\hline H.influenzae type b meningitis & 0 & 0 & 0 & 0 & 0 & 2 & 1 & 0 & 0 \\
\hline H.influenzae type b septicaemia & 0 & 0 & 0 & 0 & 0 & 0 & 1 & 0 & 0 \\
\hline H.influenzae type b infection (NOS) & 0 & 0 & 0 & 2 & 2 & 1 & 0 & 0 & 0 \\
\hline Haemolytic uraemic syndrome & 0 & 0 & 0 & 0 & 0 & 0 & 0 & 1 & 0 \\
\hline Hepatitis A* & 81 & 130 & 131 & 14 & 217 & 105 & 40 & 132 & 18 \\
\hline Hepatitis B: acute viral* & 2 & 1 & 0 & 0 & 15 & 0 & 2 & 9 & 6 \\
\hline Hepatitis B: chronic or carrier* & 21 & 6 & 13 & 0 & 396 & 100 & 21 & 567 & 20 \\
\hline Hepatitis B: unspecified* & 23 & 22 & 450 & 25 & 127 & 1024 & 40 & 152 & 13 \\
\hline Hepatitis C: acute viral* & 1 & 0 & 1 & 0 & 3 & 2 & 1 & 6 & 0 \\
\hline Hepatitis C: unspecified* & 166 & 476 & 544 & 283 & 1410 & 1242 & 435 & 1394 & 79 \\
\hline Hepatitis D: acute viral* & 0 & 0 & 0 & 0 & 0 & 0 & 0 & 0 & 0 \\
\hline Hepatitis D: unspecified* & 0 & 1 & 0 & 0 & 2 & 1 & 0 & 4 & 0 \\
\hline Hepatitis E* & 0 & 0 & 0 & 0 & 2 & 0 & 1 & 1 & 0 \\
\hline Hepatitis: acute viral (NOS) & 0 & 0 & 0 & 0 & 1 & 0 & 0 & 0 & 0 \\
\hline HIV infection* & 2 & 7 & 35 & 1 & 132 & 24 & 1 & 15 & 86 \\
\hline Legionnaires' disease* & 0 & 1 & 4 & 0 & 5 & 2 & 2 & 0 & 0 \\
\hline Leptospirosis* & 8 & 3 & 0 & 2 & 1 & 1 & 0 & 0 & 2 \\
\hline Listeriosis* & 1 & 0 & 1 & 1 & 3 & 2 & 1 & 4 & 0 \\
\hline Malaria* & 6 & 7 & 48 & 6 & 26 & 18 & 5 & 30 & 0 \\
\hline Measles & 13 & 2 & 23 & 13 & 19 & 22 & 13 & 41 & 4 \\
\hline Meningococcal infections (total) & 6 & 6 & 15 & 2 & 24 & 21 & 23 & 31 & 2 \\
\hline Meningococcal meningitis & 2 & 3 & 11 & 1 & 9 & 13 & 4 & 13 & 0 \\
\hline Meningococcal septicaemia & 3 & 3 & 2 & 0 & 5 & 7 & 13 & 13 & 2 \\
\hline Meningococcal infection NOS) & 1 & 0 & 2 & 1 & 10 & 1 & 6 & 5 & 0 \\
\hline Mumps* & 0 & 2 & 4 & 0 & 6 & 0 & 0 & 3 & 0 \\
\hline Mycobacterial infection: other than TB* & 6 & 13 & 61 & 9 & 60 & 41 & 3 & 33 & 11 \\
\hline Mycobacterial tuberculosis & 1 & 1 & 42 & 2 & 76 & 125 & 4 & 76 & 1 \\
\hline Pertussis & 98 & 178 & 460 & 96 & 520 & 524 & 298 & 530 & 31 \\
\hline Q Fever* & 48 & 30 & 0 & 5 & 2 & 3 & 1 & 1 & 1 \\
\hline Rubella* & 8 & 14 & 20 & 3 & 28 & 9 & 12 & 14 & 0 \\
\hline Salmonella infections (total)* & 71 & 137 & 208 & 31 & 214 & 191 & 124 & 158 & 9 \\
\hline Salmonella bovis morbificans infections* & 1 & 2 & 4 & 1 & 1 & 3 & 1 & 6 & 0 \\
\hline Salmonella typhimurium infections* & 31 & 37 & 107 & 14 & 139 & 119 & 99 & 91 & 7 \\
\hline Salmonella infections (NOS)* & 39 & 98 & 97 & 16 & 74 & 69 & 24 & 61 & 2 \\
\hline Syphilis & 31 & 15 & 28 & 1 & 156 & 70 & 3 & 57 & 7 \\
\hline Tetanus & 0 & 1 & 0 & 0 & 0 & 0 & 0 & 0 & 0 \\
\hline Typhoid and paratyphoid* & 1 & 1 & 6 & 0 & 4 & 5 & 0 & 6 & 0 \\
\hline
\end{tabular}

* lab-confirmed cases only NOS $=$ Not Otherwise Specified

Area health service population estimates 1997:

NEA = New England Area $(189$ 168) $\quad$ NRA $=$ Northern Rivers Area $\quad(263$ 238) $\quad$ NSA $=$ Northern Sydney Area (746 973)

$\mathrm{SA}=$ Southern Area

$\left(\begin{array}{ll}187 & 252\end{array}\right) \quad$ SES = South Eastern Sydney $(722$ 579) $\quad$ SWS = South Western Sydney (729 364)

WEN $=$ Wentworth Area

(307 369) $\quad$ WSA $=$ Western Sydney Area $(647$ 706) $\quad$ NOS $=$ Area Not Stated 


\section{TABLE 6}

\section{DISEASE NOTIFICATIONS BY AGE AND SEX, NSW, 1997}

\begin{tabular}{|c|c|c|c|c|c|c|c|c|c|c|}
\hline \multirow[b]{2}{*}{ Conditions } & \multicolumn{3}{|c|}{$0-4$ yrs } & \multicolumn{3}{|c|}{$5-24$ yrs } & \multicolumn{4}{|c|}{$25-44$ yrs } \\
\hline & M & $\mathrm{F}$ & $\mathrm{U}$ & M & $\mathrm{F}$ & $\mathrm{U}$ & M & $\mathrm{F}$ & $\mathrm{T}$ & $\mathrm{U}$ \\
\hline Adverse event after immunisation & 26 & 23 & 0 & 1 & 0 & 0 & 0 & 0 & 0 & 0 \\
\hline AIDS & 0 & 1 & 0 & 5 & 4 & 0 & 109 & 2 & 0 & 0 \\
\hline Arboviral: Barmah virus infections* & 2 & 1 & 0 & 10 & 7 & 0 & 41 & 37 & 0 & 0 \\
\hline Arboviral: Ross river virus infections* & 6 & 4 & 0 & 91 & 123 & 1 & 358 & 353 & 0 & 3 \\
\hline Arboviral: Other Infections* & 0 & 0 & 0 & 1 & 4 & 0 & 5 & 7 & 0 & 0 \\
\hline Blood lead level $>=15 \mathrm{ug} / \mathrm{dl}$ (a) & 9 & 6 & 0 & 75 & 4 & 1 & 352 & 7 & 0 & 0 \\
\hline Brucellosis* & 0 & 0 & 0 & 0 & 0 & 0 & 0 & 0 & 0 & 0 \\
\hline Cholera* & 0 & 0 & 0 & 0 & 0 & 0 & 0 & 0 & 0 & 0 \\
\hline Cryptosporidiosis* & 46 & 48 & 0 & 14 & 31 & 0 & 10 & 4 & 0 & 0 \\
\hline Food-borne illness (NOS) & 11 & 9 & 0 & 28 & 29 & 0 & 59 & 62 & 0 & 0 \\
\hline Gastroenteritis (institution) & 63 & 65 & 5 & 11 & 46 & 0 & 27 & 98 & 0 & 0 \\
\hline Gonorrhoea* & 0 & 0 & 0 & 131 & 48 & 0 & 384 & 23 & 0 & 1 \\
\hline Invasive $H$. influenzae type $\mathrm{b}$ infections (total) & 4 & 4 & 0 & 1 & 3 & 0 & 1 & 1 & 0 & 0 \\
\hline H. influenzae type b epiglottitis & 2 & 0 & 0 & 1 & 1 & 0 & 0 & 1 & 0 & 0 \\
\hline H. influenzae type b meningitis & 0 & 2 & 0 & 0 & 1 & 0 & 0 & 0 & 0 & 0 \\
\hline H. influenzae type b septicaemia & 0 & 0 & 0 & 0 & 0 & 0 & 1 & 0 & 0 & 0 \\
\hline H. influenzae type b infection (NOS) & 2 & 2 & 0 & 0 & 1 & 0 & 0 & 0 & 0 & 0 \\
\hline Haemolytic uraemic syndrome & 0 & 1 & 0 & 0 & 0 & 0 & 0 & 0 & 0 & 0 \\
\hline Hepatitis A* & 36 & 28 & 0 & 283 & 223 & 1 & 439 & 227 & 0 & 1 \\
\hline Hepatitis B: acute viral* & 0 & 0 & 0 & 12 & 8 & 0 & 14 & 11 & 0 & 0 \\
\hline Hepatitis B: chronic or carrier* (b) & 11 & 3 & 0 & 124 & 128 & 4 & 377 & 315 & 0 & 15 \\
\hline Hepatitis B: unspecified* & 6 & 10 & 1 & 269 & 278 & 6 & 875 & 736 & 0 & 13 \\
\hline Hepatitis C: acute viral* & 0 & 0 & 0 & 3 & 3 & 0 & 10 & 3 & 0 & 0 \\
\hline Hepatitis C: unspecified* (c) & 62 & 48 & 6 & 845 & 598 & 14 & 3992 & 2045 & 1 & 60 \\
\hline Hepatitis D: acute viral* & 0 & 0 & 0 & 1 & 0 & 0 & 2 & 0 & 0 & 0 \\
\hline Hepatitis D: unspecified* & 0 & 0 & 0 & 0 & 0 & 0 & 6 & 1 & 0 & 0 \\
\hline Hepatitis $E^{*}$ & 0 & 0 & 0 & 3 & 0 & 0 & 1 & 1 & 0 & 0 \\
\hline Hepatitis: acute viral (NOS) & 0 & 0 & 0 & 0 & 0 & 0 & 1 & 0 & 0 & 0 \\
\hline HIV infection* (d) & 9 & 3 & 1 & 29 & 8 & 2 & 236 & 17 & 2 & 13 \\
\hline Legionnaires' disease* & 0 & 0 & 0 & 2 & 0 & 0 & 4 & 1 & 0 & 0 \\
\hline Leptospirosis* & 0 & 0 & 0 & 8 & 1 & 0 & 10 & 4 & 0 & 0 \\
\hline Listeriosis* & 0 & 1 & 0 & 0 & 1 & 0 & 1 & 2 & 0 & 0 \\
\hline Malaria* (e) & 2 & 0 & 0 & 28 & 13 & 2 & 70 & 30 & 0 & 2 \\
\hline Measles & 75 & 50 & 1 & 50 & 67 & 0 & 6 & 12 & 0 & 0 \\
\hline Meningococcal infections (total) & 46 & 35 & 0 & 51 & 55 & 0 & 9 & 7 & 0 & 0 \\
\hline Meningococcal meningitis & 26 & 10 & 0 & 25 & 29 & 0 & 6 & 5 & 0 & 0 \\
\hline Meningococcal septicaemia & 11 & 11 & 0 & 18 & 14 & 0 & 3 & 0 & 0 & 0 \\
\hline Meningococcal infection (NOS) & 9 & 14 & 0 & 8 & 12 & 0 & 0 & 2 & 0 & 0 \\
\hline Mumps* & 0 & 0 & 0 & 6 & 13 & 0 & 1 & 6 & 0 & 1 \\
\hline Mycobacterial infection: other than TB* & 16 & 20 & 1 & 5 & 2 & 0 & 49 & 21 & 0 & 0 \\
\hline Mycobacterial tuberculosis & 6 & 3 & 0 & 27 & 30 & 0 & 100 & 90 & 0 & 0 \\
\hline Pertussis & 251 & 284 & 2 & 1072 & 1165 & 4 & 358 & 567 & 0 & 5 \\
\hline Q Fever* & 0 & 0 & 0 & 48 & 5 & 0 & 106 & 19 & 0 & 0 \\
\hline Rubella* & 8 & 4 & 0 & 56 & 29 & 0 & 23 & 21 & 0 & 0 \\
\hline Salmonella infections (total)* & 351 & 261 & 2 & 261 & 242 & 1 & 178 & 169 & 0 & 0 \\
\hline Salmonella bovis morbificans infections* & 7 & 7 & 0 & 5 & 1 & 0 & 2 & 2 & 0 & 0 \\
\hline Salmonella typhimurium infections* & 195 & 148 & 1 & 174 & 158 & 1 & 80 & 83 & 0 & 0 \\
\hline Salmonella infections (NOS)* & 149 & 106 & 1 & 82 & 83 & 0 & 96 & 84 & 0 & 0 \\
\hline Syphilis & 3 & 2 & 1 & 22 & 61 & 1 & 135 & 130 & 0 & 2 \\
\hline Tetanus & 0 & 0 & 0 & 0 & 0 & 0 & 0 & 0 & 0 & 0 \\
\hline Typhoid and paratyphoid* & 0 & 1 & 0 & 9 & 8 & 0 & 6 & 4 & 0 & 0 \\
\hline
\end{tabular}

* Laboratory-confirmed cases only

NOS $=$ Not Otherwise Specified
(a) $=2$ cases unknown age/sex
(b) $=7$ cases unknown age/sex
(c) $=8$ cases unknown age/sex
(d) $=6$ cases unknown age/sex
(e) $=1$ case unknown age/sex 


\section{TABLE 6}

\section{DISEASE NOTIFICATIONS BY AGE AND SEX, NSW, 1997 continued}

\begin{tabular}{|c|c|c|c|c|c|c|}
\hline Conditions & M & $\begin{array}{c}45-64 \\
F\end{array}$ & U & M & $>=65 \mathrm{yrs}$ & U \\
\hline Adverse event after immunisation & 0 & 0 & 0 & 0 & 0 & 0 \\
\hline AIDS & 41 & 0 & 0 & 1 & 0 & 0 \\
\hline Arboviral: Barmah virus infections* & 38 & 31 & 0 & 16 & 7 & 0 \\
\hline Arboviral: Ross river virus infections* & 262 & 277 & 2 & 59 & 69 & 1 \\
\hline Arboviral: Other Infections* & 5 & 9 & 0 & 2 & 2 & 0 \\
\hline Blood lead level >= 15ug/dl (a) & 180 & 3 & 0 & 9 & 1 & 0 \\
\hline Brucellosis* & 1 & 1 & 0 & 0 & 1 & 0 \\
\hline Cholera* & 1 & 0 & 0 & 0 & 0 & 0 \\
\hline Cryptosporidiosis* & 2 & 1 & 0 & 2 & 1 & 0 \\
\hline Food-borne illness (NOS) & 28 & 29 & 0 & 5 & 11 & 0 \\
\hline Gastroenteritis (institution) & 27 & 68 & 1 & 121 & 412 & 2 \\
\hline Gonorrhoea* & 46 & 0 & 0 & 4 & 1 & 0 \\
\hline Invasive $H$. influenzae type b infections (total) & 2 & 0 & 0 & 1 & 1 & 0 \\
\hline H. influenzae type b epiglottitis & 0 & 0 & 0 & 0 & 0 & 0 \\
\hline$H$. influenzae type b meningitis & 0 & 0 & 0 & 0 & 0 & 0 \\
\hline H. influenzae type b septicaemia & 0 & 0 & 0 & 0 & 0 & 0 \\
\hline$H$. influenzae type b infection (NOS) & 2 & 0 & 0 & 1 & 1 & 0 \\
\hline Haemolytic uraemic syndrome & 1 & 1 & 0 & 0 & 0 & 0 \\
\hline Hepatitis $A^{*}$ & 108 & 62 & 0 & 17 & 27 & 0 \\
\hline Hepatitis B: acute viral* & 2 & 1 & 0 & 1 & 1 & 0 \\
\hline Hepatitis B: chronic or carrier* (b) & 126 & 78 & 2 & 27 & 23 & 1 \\
\hline Hepatitis B: unspecified* & 313 & 177 & 2 & 73 & 56 & 1 \\
\hline Hepatitis C: acute viral* & 0 & 1 & 0 & 0 & 0 & 0 \\
\hline Hepatitis C: unspecified* (c) & 611 & 305 & 1 & 159 & 160 & 1 \\
\hline Hepatitis D: acute viral* & 0 & 0 & 0 & 0 & 0 & 0 \\
\hline Hepatitis D: unspecified* & 1 & 1 & 0 & 0 & 0 & 0 \\
\hline Hepatitis E* & 1 & 0 & 0 & 0 & 0 & 0 \\
\hline Hepatitis: acute viral (NOS) & 0 & 0 & 0 & 0 & 0 & 0 \\
\hline HIV infection* (d) & 64 & 2 & 2 & 4 & 0 & 0 \\
\hline Legionnaires' disease* & 4 & 3 & 0 & 12 & 7 & 0 \\
\hline Leptospirosis* & 4 & 3 & 0 & 4 & 0 & 0 \\
\hline Listeriosis* & 3 & 1 & 0 & 12 & 2 & 0 \\
\hline Malaria* (e) & 22 & 9 & 2 & 9 & 2 & 0 \\
\hline Measles & 1 & 2 & 0 & 0 & 0 & 0 \\
\hline Meningococcal infections (total) & 6 & 9 & 0 & 1 & 3 & 0 \\
\hline Meningococcal meningitis & 2 & 5 & 0 & 1 & 1 & 0 \\
\hline Meningococcal septicaemia & 3 & 3 & 0 & 0 & 2 & 0 \\
\hline Meningococcal infection (NOS) & 1 & 1 & 0 & 0 & 0 & 0 \\
\hline Mumps* & 1 & 0 & 0 & 1 & 0 & 0 \\
\hline Mycobacterial infection: other than TB* & 41 & 36 & 0 & 95 & 80 & 3 \\
\hline Mycobacterial tuberculosis & 52 & 45 & 0 & 63 & 45 & 0 \\
\hline Pertussis & 207 & 261 & 1 & 66 & 87 & 0 \\
\hline Q Fever* & 50 & 16 & 0 & 17 & 4 & 0 \\
\hline Rubella* & 1 & 7 & 0 & 2 & 3 & 0 \\
\hline Salmonella infections (total)* & 80 & 91 & 0 & 38 & 55 & 0 \\
\hline Salmonella bovis morbificans infections* & 1 & 2 & 0 & 0 & 0 & 0 \\
\hline Salmonella typhimurium infections* & 39 & 35 & 0 & 13 & 22 & 0 \\
\hline Salmonella infections (NOS)* & 40 & 54 & 0 & 25 & 33 & 0 \\
\hline Syphilis & 114 & 26 & 2 & 46 & 25 & 0 \\
\hline Tetanus & 0 & 1 & 0 & 0 & 2 & 0 \\
\hline Typhoid and paratyphoid* & 3 & 1 & 0 & 1 & 0 & 0 \\
\hline
\end{tabular}

* Laboratory-confirmed cases only

NOS $=$ Not Otherwise Specified
(a) $=2$ cases unknown age/sex
(b) $=7$ cases unknown age/sex
(c) $=8$ cases unknown age/sex
(d) $=6$ cases unknown age/sex
(e) $=1$ case unknown age/sex 
TABLE 7

DISEASE NOTIFICATIONS BY MONTH OF ONSET, NSW, 1997

\begin{tabular}{|c|c|c|c|c|c|c|c|c|c|c|c|c|}
\hline Conditions & JAN & FEB & MAR & APR & MAY & JUN & JUL & AUG & SEP & OCT & NOV & DEC \\
\hline Adverse event after immunisation & 4 & 4 & 0 & 2 & 2 & 0 & 6 & 7 & 8 & 1 & 5 & 11 \\
\hline AIDS & 22 & 16 & 12 & 16 & 14 & 16 & 5 & 19 & 14 & 6 & 9 & 14 \\
\hline Arboviral: Barmah virus infections* & 22 & 17 & 25 & 31 & 32 & 12 & 9 & 4 & 11 & 7 & 6 & 14 \\
\hline Arboviral: Ross river virus infections* & 173 & 203 & 201 & 413 & 348 & 128 & 33 & 24 & 18 & 24 & 19 & 26 \\
\hline Arboviral: Other Infections* & 9 & 7 & 5 & 4 & 2 & 1 & 1 & 0 & 2 & 0 & 1 & 3 \\
\hline Blood lead level $>=15 \mathrm{ug} / \mathrm{dl}$ & 132 & 52 & 50 & 25 & 27 & 23 & 41 & 77 & 43 & 67 & 70 & 46 \\
\hline Brucellosis* & 0 & 0 & 1 & 0 & 1 & 0 & 0 & 0 & 0 & 0 & 1 & 0 \\
\hline Cholera* & 0 & 0 & 0 & 0 & 0 & 1 & 0 & 0 & 0 & 0 & 0 & 0 \\
\hline Cryptosporidiosis* & 11 & 15 & 7 & 8 & 4 & 5 & 3 & 3 & 2 & 4 & 24 & 73 \\
\hline Food-borne illness (NOS) & 23 & 1 & 12 & 11 & 3 & 7 & 10 & 4 & 75 & 5 & 20 & 101 \\
\hline Gastroenteritis (in an institution) & 86 & 75 & 47 & 33 & 32 & 42 & 225 & 65 & 66 & 146 & 129 & 0 \\
\hline Gonorrhoea* & 55 & 42 & 62 & 56 & 56 & 58 & 68 & 56 & 59 & 42 & 43 & 42 \\
\hline Invasive $H$. influenzae type b & & & & & & & & & & & & \\
\hline infections (total) & 4 & 1 & 0 & 0 & 3 & 1 & 1 & 2 & 3 & 1 & 1 & 1 \\
\hline H. influenzae type b epiglottitis & 1 & 1 & 0 & 0 & 2 & 0 & 1 & 0 & 0 & 0 & 0 & 0 \\
\hline H. influenzae type b meningitis & 0 & 0 & 0 & 0 & 0 & 0 & 0 & 2 & 0 & 1 & 0 & 0 \\
\hline H. influenzae type b septicaemia & 0 & 0 & 0 & 0 & 1 & 0 & 0 & 0 & 0 & 0 & 0 & 0 \\
\hline H. influenzae type b infection (NOS) & 3 & 0 & 0 & 0 & 0 & 1 & 0 & 0 & 3 & 0 & 1 & 1 \\
\hline Haemolytic uraemic syndrome & 0 & 0 & 0 & 0 & 0 & 0 & 0 & 1 & 0 & 0 & 1 & 1 \\
\hline Hepatitis $A^{*}$ & 160 & 327 & 85 & 85 & 101 & 140 & 94 & 90 & 67 & 94 & 88 & 124 \\
\hline Hepatitis B: acute viral* & 6 & 4 & 6 & 7 & 5 & 7 & 3 & 2 & 4 & 2 & 0 & 4 \\
\hline Hepatitis B: chronic or carrier* & 101 & 96 & 98 & 127 & 96 & 84 & 121 & 122 & 109 & 87 & 100 & 104 \\
\hline Hepatitis B: unspecified* & 230 & 234 & 240 & 251 & 258 & 207 & 277 & 218 & 213 & 221 & 246 & 223 \\
\hline Hepatitis C: acute viral* & 0 & 0 & 2 & 2 & 1 & 3 & 0 & 1 & 0 & 0 & 5 & 6 \\
\hline Hepatitis C: unspecified* & 735 & 804 & 707 & 726 & 733 & 688 & 770 & 765 & 849 & 751 & 726 & 688 \\
\hline Hepatitis D: acute viral* & 0 & 1 & 2 & 0 & 0 & 0 & 0 & 0 & 0 & 0 & 0 & 0 \\
\hline Hepatitis D: unspecified* & 1 & 2 & 0 & 1 & 0 & 1 & 0 & 1 & 0 & 2 & 0 & 1 \\
\hline Hepatitis $\mathrm{E}^{*}$ & 2 & 0 & 2 & 0 & 0 & 0 & 0 & 2 & 0 & 0 & 0 & 0 \\
\hline Hepatitis: acute viral (NOS) & 0 & 0 & 0 & 0 & 0 & 1 & 0 & 0 & 0 & 0 & 0 & 0 \\
\hline HIV infection* & 39 & 29 & 33 & 38 & 32 & 28 & 30 & 32 & 37 & 31 & 35 & 34 \\
\hline Legionnaires' disease* & 1 & 4 & 3 & 2 & 5 & 6 & 5 & 1 & 1 & 1 & 1 & 3 \\
\hline Leptospirosis* & 1 & 3 & 1 & 5 & 5 & 3 & 3 & 1 & 3 & 2 & 4 & 3 \\
\hline Listeriosis* & 3 & 1 & 4 & 0 & 2 & 0 & 3 & 2 & 3 & 1 & 1 & 3 \\
\hline Malaria* & 22 & 11 & 14 & 21 & 21 & 17 & 14 & 14 & 20 & 12 & 8 & 18 \\
\hline Measles & 10 & 14 & 14 & 6 & 20 & 23 & 30 & 23 & 16 & 43 & 43 & 22 \\
\hline Meningococcal infections (total) & 13 & 4 & 6 & 20 & 13 & 16 & 34 & 32 & 28 & 23 & 16 & 17 \\
\hline Meningococcal meningitis & 7 & 2 & 3 & 11 & 9 & 5 & 18 & 19 & 15 & 11 & 5 & 5 \\
\hline Meningococcal septicaemia & 5 & 2 & 3 & 4 & 2 & 9 & 7 & 6 & 10 & 6 & 5 & 6 \\
\hline Meningococcal infection (NOS) & 1 & 0 & 0 & 5 & 2 & 2 & 9 & 7 & 3 & 6 & 6 & 6 \\
\hline Mumps* & 6 & 1 & 11 & 1 & 1 & 0 & 2 & 1 & 1 & 1 & 3 & 1 \\
\hline Mycobacterial infection: other than TB* & 32 & 27 & 26 & 43 & 33 & 26 & 45 & 27 & 32 & 26 & 31 & 23 \\
\hline Mycobacterial tuberculosis & 53 & 33 & 44 & 31 & 41 & 31 & 31 & 36 & 51 & 26 & 57 & 27 \\
\hline Pertussis & 263 & 218 & 179 & 159 & 198 & 237 & 361 & 375 & 366 & 636 & 717 & 627 \\
\hline Q Fever* & 28 & 16 & 14 & 23 & 35 & 27 & 26 & 19 & 17 & 21 & 21 & 18 \\
\hline Rubella* & 25 & 24 & 15 & 6 & 11 & 14 & 15 & 13 & 7 & 9 & 7 & 9 \\
\hline Salmonella infections (total)* & 163 & 150 & 215 & 162 & 120 & 74 & 88 & 91 & 146 & 180 & 124 & 219 \\
\hline Salmonella bovis morbificans infections* & $* 1$ & 2 & 2 & 3 & 2 & 1 & 0 & 2 & 5 & 1 & 4 & 4 \\
\hline Salmonella typhimurium infections* & 77 & 81 & 110 & 85 & 51 & 30 & 57 & 55 & 86 & 119 & 67 & 132 \\
\hline Salmonella infections (NOS)* & 85 & 67 & 103 & 74 & 67 & 43 & 31 & 34 & 55 & 60 & 53 & 83 \\
\hline Syphilis & 51 & 63 & 58 & 42 & 60 & 42 & 49 & 53 & 32 & 40 & 37 & 44 \\
\hline Tetanus & 0 & 0 & 0 & 0 & 1 & 0 & 1 & 0 & 0 & 0 & 0 & 1 \\
\hline Typhoid and paratyphoid* & 9 & 3 & 4 & 4 & 1 & 0 & 3 & 1 & 1 & 1 & 0 & 6 \\
\hline
\end{tabular}

* Laboratory-confirmed cases only

NOS $=$ Not otherwise specified 